\title{
Uncommon Manifestations of Acute Lymphoblastic Leukaemia (ALL)
}

\author{
SUNIL GOMBER, MANISH NARANG AND UPREET DHALIWAL
}

\begin{abstract}
Unusual manifestations often lead to misdiagnosis of patients with acute lymphoblastic leukemia (ALL). We describe two children with ALL having unusual associations. The first case presented with pseudohypopyon after 18 months into her maintenance therapy whereas the other case masqueraded as juvenile rheumatoid arthritis at presentation.
\end{abstract}

\section{INTRODUCTION}

Acute lymphoblastic leukaemia (ALL) represents about $75 \%$ of all leukaemia cases in children. Mostly, the children present with pallor, bleeding manifestations and hepatosplenomegaly. However, at times, manifestations can be unusual, making it difficult to attribute it to leukaemia. The present communication is regarding uncommon manifestations, in 2 children suffering from ALL, one during maintenance therapy and the other at presentation.

Case 1: A 5 year old female child presented with complaints of low grade, intermittent fever for 8 months and progressive pallor for last 2 months. On examination child was pale with generalised lymphadenopathy and hepatosplenomegaly. Peripheral smear revealed $10 \%$ lymphoblasts; bone marrow aspiration was suggestive of acute lymphoblastic leukaemia (ALL). Cytochemistry showed PAS positive,

Department of Pediatrics (Sunil Gomber \& Manish Narang) and Opthalmology (Upreet Dhaliwal) University College of Medical Sciences and GTB Hospital Delhi

Correspondence to: MANISH NARANG

Email: manish_2710@yahoo.com
Sudan Black negative cells suggestive of ALL. He was treated with MCP- 841 protocol. After 1/1/2 year of maintenance therapy, she developed pain and redness in the right eye. A clinical diagnosis of pseudohypopyon (Fig. 1) was made and

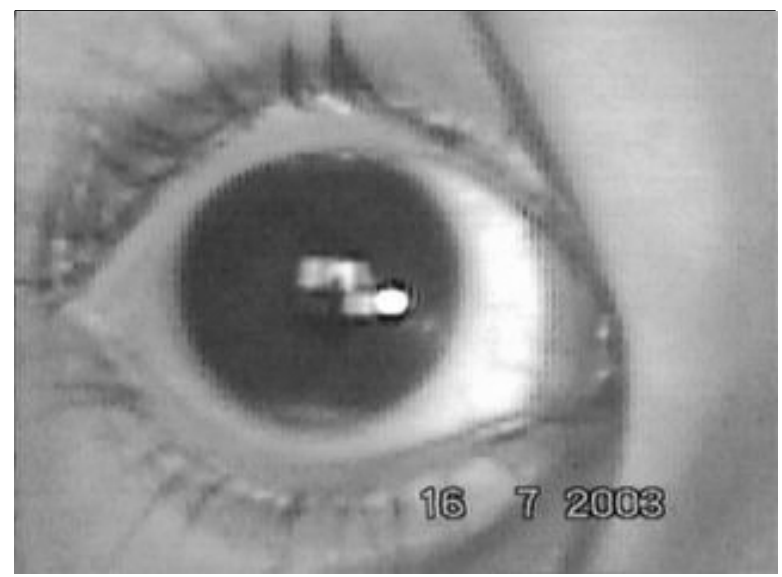

Figure 1: Pseudohypopyon in a child with ALL.

possibility of ocular recurrence was suggested. Subsequent bone marrow aspiration and CSF done for malignant cells was negative for blasts. The pseudohypopyon disappeared on topical steroid and child remained well. However, she had a recurrence of pseudohypopyon 6 months later. The aspiration cytology from the anterior chamber of the affected eye revealed lymphoblasts with no evidence of central nervous system disease on CT scan of brain and CSF examination. The child was discharged on standard MCP- 841 protocol and was advised to follow up with oncology department. However, she was lost to follow up and was brought in a moribund condition 4 months later and was found to have frank central nervous system relapse. She expired 7 days later. 
Case 2: An 11 year old male patient presented with complaints of joint pain for last 6 months which started from right knee and spread to other major joints. Pain was associated with swelling and redness of joints and mild fever. There was no history of morning stiffness, rashes and bleeding from any site. There was no family history of similar complaint. On examination the child was found to be pale with joint swelling, redness, tenderness and restriction of movements. Liver was $1.5 \mathrm{~cm}$ below costal margin, firm and spleen was not palpable. Provisional diagnosis of pauciarticular rheumatoid arthritis was made. Child was put on aspirin $(100 \mathrm{mg} / \mathrm{kg} /$ day $)$ and investigated on lines of rheumatoid arthritis. Child failed to respond to aspirin. Haemogram done at this point of time revealed $70 \%$ blasts. Subsequently bone marrow aspiration and cytochemical staining confirmed ALL. Child was put on induction regimen of ALL using intrathecal methotrexate, IV vincristine and prednisolone. There was disappearance of pain, swelling, redness and restriction of movements. The child was discharged after induction therapy and was advised for follow up for maintenance therapy as per MCP 841 protocol.

\section{DISCUSSION}

These two cases demonstrate uncommon manifestations of ALL making it difficult for a general physician/ paediatrician to clinch the diagnosis. The first case presented only with relapse of leukaemia in the iris suggested by the presence of lymphoblasts in the anterior chamber without any other CNS involvement. The anterior segment is an uncommon site of extramedullary relapse, accounting for between $0.5 \%$ and $2.6 \%$ of all relapses in large published series of acute lymphoblastic leukaemia (ALL). ${ }^{1}$ The presenting symptoms of anterior segment relapse are generally non-specific, these include redness, epiphora, photophobia, ocular pain, change in colour and appearance of iris and rarely visual loss. The clinical findings are very varied, with iritis and hypopyon being the most common. High risk period for anterior chamber leukaemic relapse is first few months following maintenance therapy withdrawl., ${ }^{2,3}$ Eyes may remain a sanctuary site for leukaemic cells during treatment, as they are routinely shielded during central nervous system irradiation and chemotherapy agents do not penetrate the eye wall. ${ }^{4}$

The diagnosis of leukemic iris infiltration is often made clinically on slit-lamp examination but should be confirmed with aqueous cytology or iris histopathology. ${ }^{5}$ In anterior segment leukemic infiltrates segmental or baggy swelling of iris is common, associated with segmental or total ciliary injection. Hypopyon and/or hyphema usually are present. The absence of posterior synechias and increased intraocular pressure are additional characteristics that differentiate anterior segment leukemic infiltrates from anterior uveitis. ${ }^{6}$ Prompt anterior chamber paracentesis and pathological studies should be done in such cases where history of leukemia even though other systemic investigations might indicate remission ${ }^{7}$. In our case, anterior chamber tap, being an invasive procedure, was not attempted on earlier two occasions as the vision was normal and the uveitis responded rapidly to topical steroid and cycloplegic therapy. Definitive local treatment is with external beam radiation. Systemic chemotherapy is not required unless there is a systemic relapse of the leukemia. Thus, Leukemic involvement of the anterior segment of the eye should be suspected in all cases of uveitis that are refractory to treatment, and particularly when a hypopyon uveitis develops in a child. When the patient is already a diagnosed case of leukemia, the occurrence of uveitis is very likely to be a sign of CNS relapse..$^{5-7}$

Musculoskeletal symptoms related to ALL are bone pains, abnormal gait, kyphosis, lordosis, unusual fracture and decreased or delayed linear growth. Bone pain is caused by pressure from infiltration of leukaemic cells into medullary cavity or under the periosteum, while joint pain 
is usually thought to be referred from lesions of periosteum. ${ }^{8}$ Joint pain is frequently migrating in character $^{9}$ sometimes leading to initial presumptive diagnosis of rheumatic fever, juvenile rheumatoid arthritis (as in present case-2) or septic arthritis. A markedly elevated WBC count $>50,000$ occurs in about $20 \%$ of patients. Bone pain is present in less than one third of children who had leukaemia of any type. ${ }^{10}$ In a review by Silverstein et al, 29 patients referred for presumed rheumatological illness over a 13 year period $(0.4 \%$ of all referrals) were eventually given a diagnosis of malignancy most often, ALL ${ }^{9}$. Thrombocytopenia is rare in persons with JRA; its presence, although it raises the possibility of SLE, also suggests the possibility of leukemia. The differential count in JRA often demonstrates a relative lymphopenia, presumably because of migration of activated lymphocytes from circulation into synovium. Lymphocytosis is uncharacteristic of JRA and raises the possibility of leukemia, particularly when neutropenia is present. Therefore, clinicians should maintain high index of suspicion for malignancy when a child complains of chronic persistent non-articular bone or back pain.

\section{REFERENCES:}

1. Bunin N, Rivera G, Goode F, Hustu HO. Ocular relapse in the anterior chamber in childhood acute lymphoblastic leukaemia. J Clin Oncol 1987;5:299-303.

2. Novakovic P, Kelly S, Taylor D. Childhood leukaemia: relapse in the anterior segment of the eyes. $\mathrm{Br} J$ Ophthalmol 1989;73:354-359.

3. Maclean H, Clarke MP, Strong NP, Kernahan J, Ashraf $S$. Primary ocular relapse in acute lymphoblastic leukaemia. Eye, 1996;10:719- 722.

4. Ninane $J$, Taylor $D$, Pay $S$. The eyes as a sanctuary site in acute lymphoblastic leukaemia. Lancet, 1980;1:452-453.

5. Patel SV, Herman DC, Anderson PM, Al-Zein NJ, Buettner $H$. Iris and anterior chamber involvement in acute lymphoblastic leukemia. J Pediatr Hematol Oncol. 2003;25:653-656.

6. Badeeb O, Anwar M, Farwan K, Tashkandi I, Omar $A$, Marzouki A. Leukemic infiltrate versus anterior uveitis. Ann Ophthalmol. 1992;24:295-298.

7. Wadhwa $N$, Vohra $R$, Shrey D, Iyer VK, Garg $S$.Unilateral hypopyon in a child as a first and sole presentation in relapsing acute lymphoblastic leukemia. Indian J Ophthalmol. 2007;55:223-224.

8. Gallagher DJ, Phillips DJ, Heinrich SD. Orthopedic manifestations of acute pediatric leukemia. Orthop Clin North Am 1996;27:635-644.

9. Silverstein MN, Kelly PJ. Leukemia with osteoarticular symptoms and signs. Ann Intern Med 1963:59:637-645.

10. Pui CH: Childhood leukemias, In: Murphy GP, Lawrence W, Lenhard RE (eds): American Cancer Society Textbook of Clinical Oncology, ed 2. Atlanta, American Cancer Society, 1995;501-523. 